\title{
Formación electroquímica de películas de polipirrol sobre Nitinol a partir de soluciones de ácido sulfosuccínico
}

\author{
Electrochemical formation of polypyrrole films on \\ Nitinol from sulfosuccinic acid solutions
}

Melisa Saugo ${ }^{1}$, Daniel Omar Flamini ${ }^{1}$,
Silvana Beatriz Saidman

\author{
${ }^{1}$ Instituto de Ingeniería Electroquímica y Corrosión (INIEC), Departamento de Ingeniería Química, Universidad Nacional \\ del Sur, Av. Alem 1253, Bahía Blanca, Buenos Aires, Argentina \\ e-mail: melisa.saugo@uns.edu.ar; dflamini@uns.edu.ar; ssaidman@criba.edu.ar
}

\section{RESUMEN}

En este trabajo se estudió la electrosíntesis de polipirrol (PPy) sobre Nitinol a partir de soluciones de ácido sulfosuccínico $(0,25 \mathrm{M})$ y pirrol $(0,25 \mathrm{M})$. El PPy fue formado potenciostáticamente en soluciones de $\mathrm{pH} 1 \mathrm{y}$ 7. Hasta donde tenemos conocimiento, es la primera vez que se realiza la electrodeposición de PPy en presencia de ácido sulfosuccinico.

La morfología de las películas se estudió mediante imágenes de microscopia de barrido electrónico, observándose la típica forma globular del PPy. También se determinó, mediante energía dispersiva de rayos X (EDS), la presencia de S lo cual confirma que el dopante fue incorporado a la matriz polimérica. Las películas formadas son muy adherentes y presentan una conductividad aceptable.

Para poder evaluar el comportamiento ante la corrosión se realizaron diferentes experiencias electroquímicas en solución de Ringer: medición del potencial de circuito abierto, diagramas de Tafel y voltametría de barrido lineal. Si bien ambos recubrimientos mejoran la protección del sustrato, las películas formadas en solución de $\mathrm{pH} 1$ ofrecen mejores resultados. El análisis de iones en solución mediante espectrometría de emisión atómica de plasma acoplado inductivo confirma que las películas son protectoras, evitando la liberación de iones $\mathrm{Ni}$ en la solución.

Palabras clave: Nitinol, Polipirrol, Acido Sulfosuccínico, Adherencia.

\section{ABSTRACT}

In this work the electrosynthesis of polypyrrole (PPy) on Nitinol from solutions of sulfosuccinic acid $(0,25$ $\mathrm{M})$ and pyrrole $(0,25 \mathrm{M})$ was studied. The PPy was formed potentiostatically in solutions of $\mathrm{pH} 1$ and 7 . As far as we know, this is the first time that the electrodeposition of PPy is carried out in the presence of sulfosuccinic acid.

The morphology of the films was studied by scanning electron microscopy images, observing the typical globular shape of PPy. The presence of S was also determined by X-ray dispersive energy (EDX), confirming that the dopant was incorporated into the polymer matrix. The films formed are very adherent and exhibit acceptable conductivity.

In order to evaluate the corrosion behaviour, different electrochemical experiments were performed in Ringer's solution: measurement of open circuit potential, Tafel plots and linear sweep voltammetry. While both coatings improve the protection of the substrate, the films formed in $\mathrm{pH} 1$ solution offer better results. The analysis of ions in solution by inductive coupled plasma atomic emission spectrometry confirms that the films are protective, preventing the release of $\mathrm{Ni}$ ions into the solution.

Keywords: Nitinol, Polypyrrole, Sulfosuccinic acid, Adherence. 
El Nitinol (NiTi) es una aleación equiatómica de Ni y Ti utilizada como biomaterial debido a sus propiedades mecánicas y su biocompatibilidad. Entre sus aplicaciones más importantes se puede citar su empleo en implantes para hueso [1] y stents cardiovasculares [2]. El mayor riesgo que presenta el uso de esta aleación es la liberación de iones $\mathrm{Ni}^{2+}$ en el cuerpo, lo cual puede ser alergénico e incluso cancerígeno [3]. Una alternativa para evitar este problema es recubrir el material con un polímero conductor que lo proteja de la corrosión. El mecanismo de protección mediante polímeros conductores es complejo y sigue aún en estudio, aunque se ha aceptado que no solo actúan como una barrera física, sino que también pueden desacelerar la disolución del metal estabilizando el potencial en la zona pasiva [4]. Una buena opción dentro de los polímeros conductores es el polipirrol (PPy), un polímero biocompatible [5] que puede ser formado electroquímicamente de una manera relativamente sencilla. Además, también se sabe que el PPy puede proteger diferentes sustratos ante la corrosión, incluyendo al NiTi, cuando es dopado con distintos aniones que son inhibidores de la corrosión [6-8]. Sin embargo, el principal problema es la adherencia del polímero al sustrato [9]. En este trabajo, se utiliza ácido sulfosuccínico (AS) durante la polimerización con el fin de incrementar la adherencia de la película formada. La fórmula estructural para este ácido se presenta en la Fig. 1. Como se puede observar, posee un grupo sulfónico, $\mathrm{SO}_{3} \mathrm{H}$, el cual actúa como fuente de protones [10]. Hasta donde tenemos conocimiento, esta es la primera vez que se utiliza este ácido durante la electrosíntesis del PPy. El uso de sulfosuccinatos (a partir de sales de diésteres del ácido sulfosuccínico) como dopantes del PPy, tal como el dioctilsulfosuccinato de sodio, sí ha sido reportado ampliamente [11-13].

Así, el objetivo de este trabajo es lograr la síntesis de películas de PPy sobre NiTi a partir de soluciones de AS de distinto $\mathrm{pH}$, con el fin de proteger al sustrato de la corrosión en solución de Ringer.

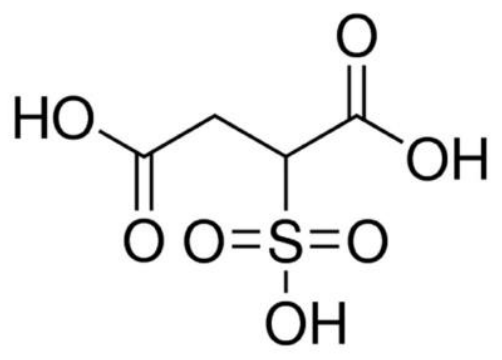

Figura 1: Fórmula estructural del ácido sulfosuccínico.

\section{MATERIALES Y MÉTODOS}

Como electrodo de trabajo (ET) se utilizó una barra cilíndrica de NiTi (composición porcentual: 55,8 Ni; 0,05 O; 0,02 C y Ti) empotrada en un cilindro de Teflón, con un área expuesta de $0,1 \mathrm{~cm}^{2}$. El ET fue pulido con lija esmeril 1200, luego desengrasado con acetona y finalmente enjuagado con agua destilada antes de cada experiencia. Los potenciales fueron medidos con respecto a un electrodo de referencia de calomel saturado (ECS). Todos los potenciales en este trabajo están referidos a ECS. Como contraelectrodo se utilizó una lámina de Pt. Para todas las experiencias electroquímicas se empleó una celda Metrohm de $20 \mathrm{~cm}^{3}$.

Las películas de PPy fueron obtenidas potenciostáticamente a partir de una solución que contiene 0,25 M de pirrol (Py) y 0,25 M AS de pH 1 y 7. En el primer caso (pH 1), se aplicó un potencial de 0,9 V(ECS) durante $600 \mathrm{~s}$, mientras que para la solución de pH 7 se aplicó un potencial de 1,0 V(ECS) durante $1375 \mathrm{~s}$, tiempo necesario para obtener películas de un espesor similar a las anteriores. Las soluciones fueron purificadas en una atmósfera saturada de gas nitrógeno a $25{ }^{\circ} \mathrm{C}$ previo a la polimerización. Todos los productos químicos utilizados fueron de grado analítico y las soluciones se prepararon utilizando agua tridestilada. El pirrol (Sigma-Aldrich) fue destilado al vacío antes de ser utilizado.

El comportamiento de los recubrimientos ante la corrosión fue evaluado mediante la variación del potencial de circuito abierto (PCA) con el tiempo y a través de medidas potenciodinámicas en solución de Ringer, la cual se usa frecuentemente para simular el medio biológico (0,147 $\mathrm{M} \mathrm{NaCl} ; 0,00432 \mathrm{M} \mathrm{CaCl}_{2}$; 0,00404 M KCl, lo cual da una concentración total de cloruro de 0,16 M [14]).

Todas las experiencias electroquímicas fueron llevadas a cabo con un potenciostato/galvanostato Autolab/PGSTAT128N. Para el estudio de la morfología de los recubrimientos se utilizó un microscopio electrónico de barrido (MEB) modelo ISI DS 130 acoplado a un analizador cuantitativo de energía dispersiva de rayos X (EDX) modelo EDAX 9600. Las concentraciones liberadas de Ni y Ti en solución fisiológica para el 
NiTi desnudo y recubierto de PPy fueron determinadas mediante espectrometría de emisión atómica de plasma acoplado inductivo (ICP-AES).

La adherencia de los recubrimientos de PPy fue determinada mediante el uso de un dinamómetro Mecmesin (BFG 50N), el cual mide la fuerza necesaria para despegar la película polimérica empleando cinta doble faz 3M (Scotch ${ }^{\circledR}$ MagicTM Tape 810).

\section{RESULTADOS Y DISCUSIÓN}

\subsection{Electrosíntesis de películas de PPy}

La electrosíntesis de PPy sobre NiTi se llevó a cabo potenciostáticamente en soluciones de AS 0,25 M de pH 1 (pH que se mide al preparar la solución) y pH 7 (alcanzado mediante el agregado de $\mathrm{NaOH}$ ). Con el propósito de encontrar las condiciones experimentales óptimas para la formación de la película, se aplicaron potenciales desde 0,6 a $0,9 \mathrm{~V}$ (ECS). Los transientes obtenidos empleando la solución de menor $\mathrm{pH}$ se muestran en la Fig. 2.

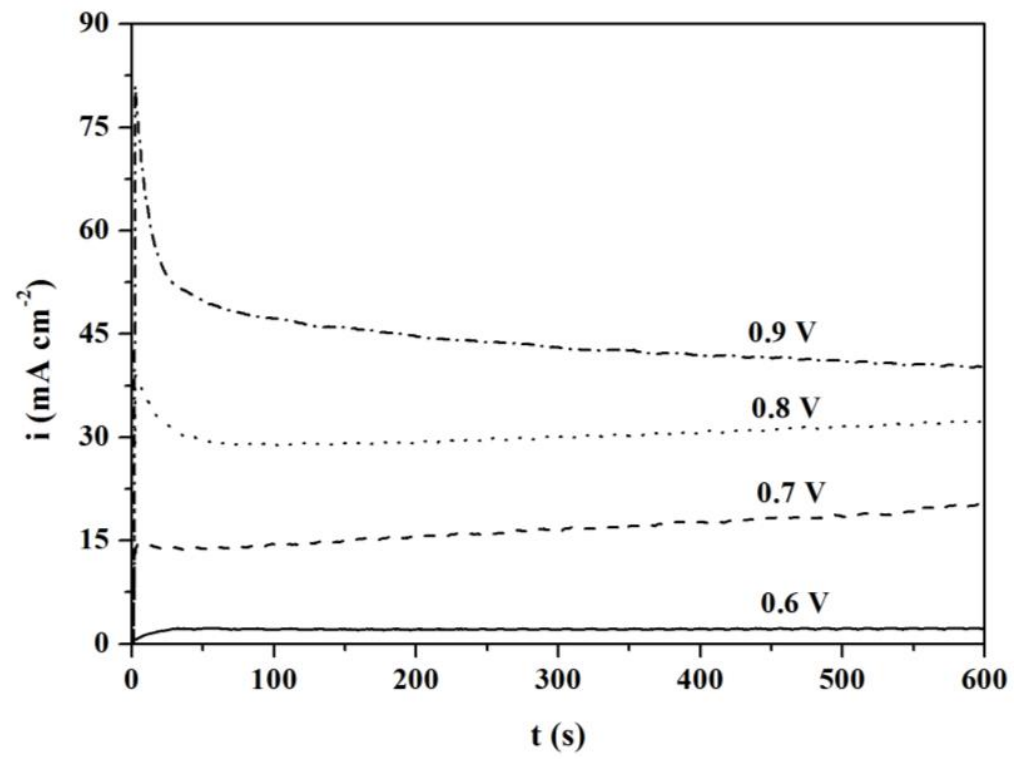

Figura 2: Curvas cronoamperométricas obtenidas para la aleación NiTi en solución 0,25 M AS + 0,25 M Py, pH 1, aplicando un potencial de 0,$6 ; 0,7 ; 0,8 ;$ y $0,9 \mathrm{~V}$ (ECS).

Para los potenciales más bajos se obtienen capas muy delgadas de polímero, las cuales pueden ser removidas con facilidad. A potenciales mayores que $0,8 \mathrm{~V}$ (ECS) sólo es posible remover el polímero mediante pulido mecánico. Se comparó también el espesor de las películas formadas. Para ésto se calculó la carga involucrada en la polimerización a partir de las curvas de la Fig. 2 y se estimó el espesor correspondiente considerando que una película de PPy es de $1 \mu \mathrm{m}$ cuando la carga involucrada en la electropolimerización es de $0,4 \mathrm{C} / \mathrm{cm}^{2}$ [15]. A partir de estos datos se decidió trabajar con las películas de PPy formadas aplicando $0,9 \mathrm{~V}(\mathrm{ECS})$ dado que, bajo estas condiciones, se forman películas adherentes y de un buen espesor $(66,4 \mu \mathrm{m})$ a un potencial relativamente bajo.

Con el fin de estudiar el efecto del pH de la solución de polimerización en las características de las películas formadas, se intentó formar el polímero en las condiciones seleccionadas previamente, pero empleando una solución de $\mathrm{pH}$ 7. Sólo fue posible formar el polímero aplicando un potencial de 1,0 V(ECS). A este potencial y durante $600 \mathrm{~s}$ se formó un recubrimiento muy delgado y de poca adherencia, por lo tanto, se aplicó el potencial durante el tiempo necesario (1375 s) para sintetizar una película de igual espesor que la formada a pH 1. El transiente se puede observar en la Fig. 3.

Con el fin de simplificar, las películas formadas a pH 1 y pH 7 serán llamadas $\mathrm{PPy}_{1}$ y $\mathrm{PPy}_{7}$, respectivamente. 


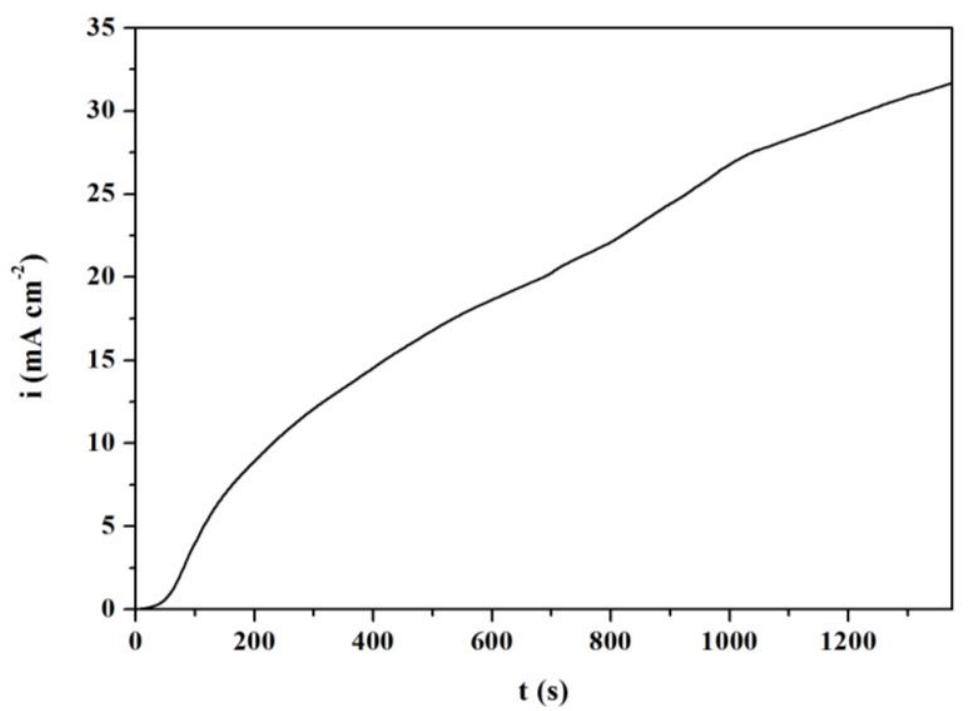

Figura 3: Curva cronoamperométrica obtenida para la aleación NiTi en solución $0,25 \mathrm{M}$ AS + 0,25 M Py, pH 7 aplicando un potencial de $1,0 \mathrm{~V}$ (ECS).

\subsection{Caracterización de las películas de PPy}

Como ya se mencionó anteriormente, las películas de $\mathrm{PPy}_{1}$ se pueden quitar solamente por pulido mecánico. Con el fin de cuantificar esta adherencia, se midió la fuerza por unidad de área necesaria para despegarlas del sustrato utilizando un dinamómetro. Para esta experiencia fue necesario utilizar como electrodo de trabajo chapas de NiTi de $1 \mathrm{~cm}^{2}$. Se realizaron 3 mediciones para cada una de las películas. Los valores promedio obtenidos fueron 4,00 $\mathrm{MPa}$ para $\mathrm{PPy}_{1}$ y 0,38 $\mathrm{MPa}$ para $\mathrm{PPy}_{7}$. Como se puede ver, $\mathrm{PPy}_{1}$ presenta una adherencia 10 veces mayor que la película formada a $\mathrm{pH}$ neutro. Se han reportado valores de fuerza de adherencia de hasta 1,54 MPa para PPy formado a partir de soluciones orgánicas [16] y de hasta 2,07 MPa [17] para PPy dopado con inhibidores de la corrosión. Se puede concluir que la muestra $\mathrm{PPy}_{1}$ presenta buena adherencia.

También se midió la conductividad de las capas de polímero, estimando el espesor de la misma en $66,37 \mu \mathrm{m}$ a partir de la carga empleada durante la electropolimerización como ya se explicó anteriormente. Los valores obtenidos, que se presentan en la Tabla 1, son mayores que los reportados, por ejemplo, para PPy dopado con ácido bórico [18].

Tabla 1: Conductividad de los distintos recubrimientos de PPy formados sobre NiTi.

\begin{tabular}{l|l}
\hline MUESTRA & $\begin{array}{l}\text { CONDUCTIVIDAD } \\
\text { [S } \mathbf{~ C M}^{-1} \text { ] }\end{array}$ \\
\hline $\mathrm{NiTi} / \mathrm{PPy}_{1}$ & 0,0013 \\
\hline $\mathrm{NiTi} / \mathrm{PPy}_{7}$ & 0,097 \\
\hline
\end{tabular}

Por último, se tomaron imágenes MEB del recubrimiento formado en solución de pH 1. Las imágenes de distintas magnificaciones se observan en la Fig. 4. 


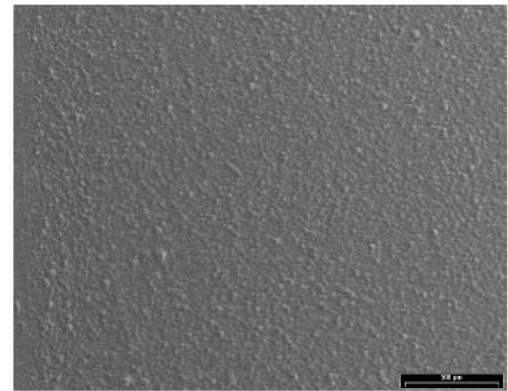

$\underline{60 X}$

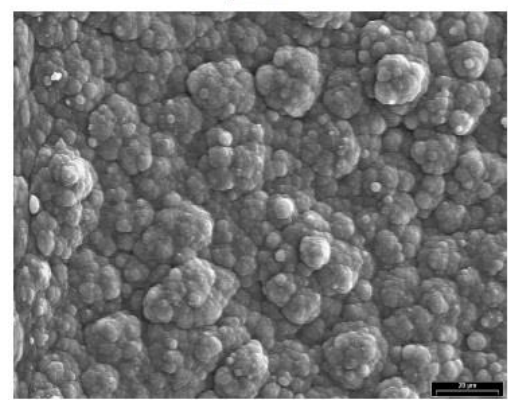

$\underline{1000 X}$

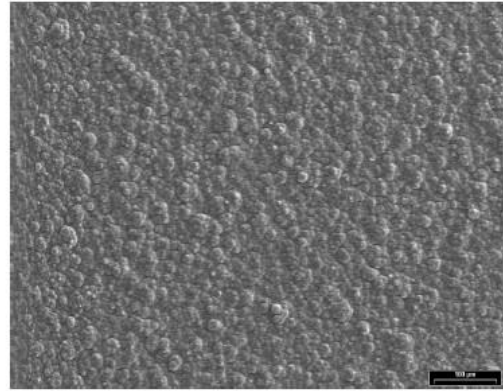

$\underline{200 X}$

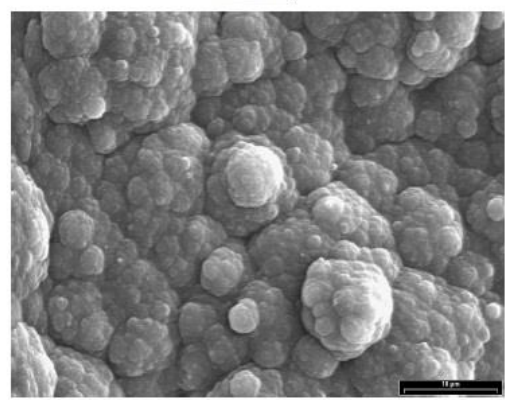

$\underline{3000 X}$

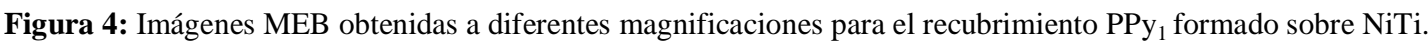

Se puede observar que el recubrimiento tiene la típica forma de coliflor que presenta el PPy [19,20]. Mediante un análisis EDS se determinó la presencia de azufre en la película (Fig. 5). En el análisis EDS no se detectaron Ni ni Ti, lo cual indica la formación de una capa compacta y gruesa de polímero.

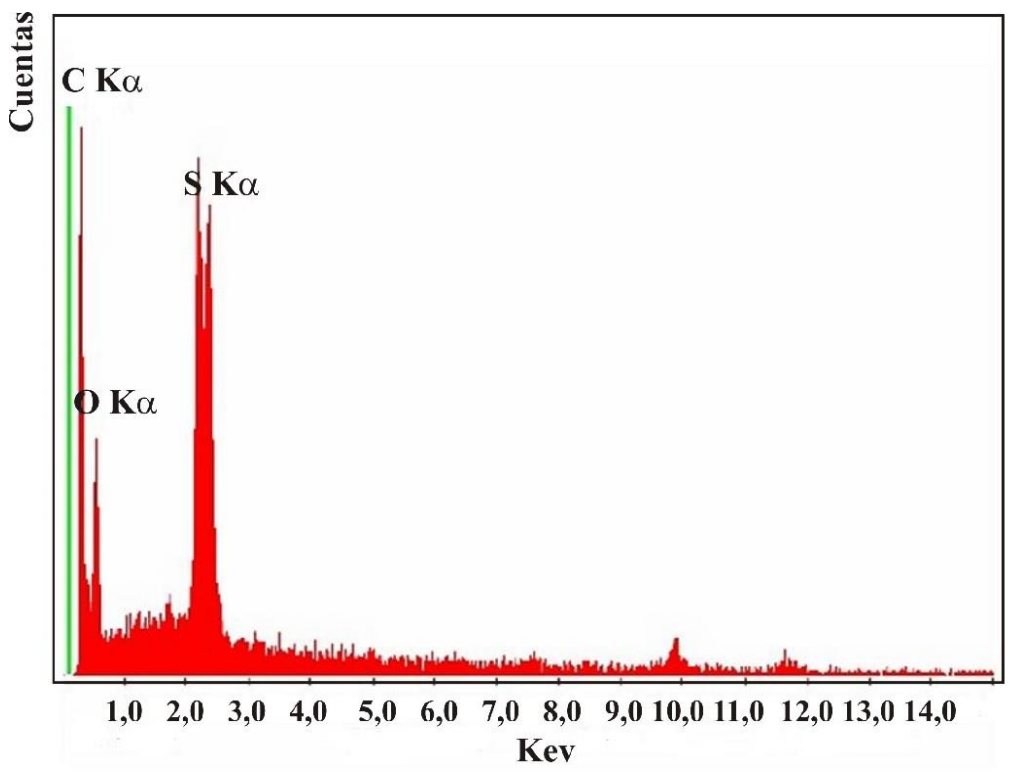

Figura 5: Espectro EDS para el recubrimiento $\mathrm{PPy}_{1}$ formado sobre NiTi.

\subsection{Protección ante la corrosión}

Uno de los principales objetivos de este trabajo es examinar el comportamiento ante la corrosión de los electrodos recubiertos. La variación del potencial a circuito abierto (PCA) en función del tiempo en un medio agresivo se puede utilizar para estimar el grado protección que el recubrimiento brinda al sustrato. Esta medición experimental fue realizada en solución de Ringer durante una semana para electrodos recubiertos con ambas películas, $\mathrm{PPy}_{1}$ y $\mathrm{PPy}_{7}$ y fueron comparados con los valores obtenidos para el NiTi desnudo (Fig. 6). 


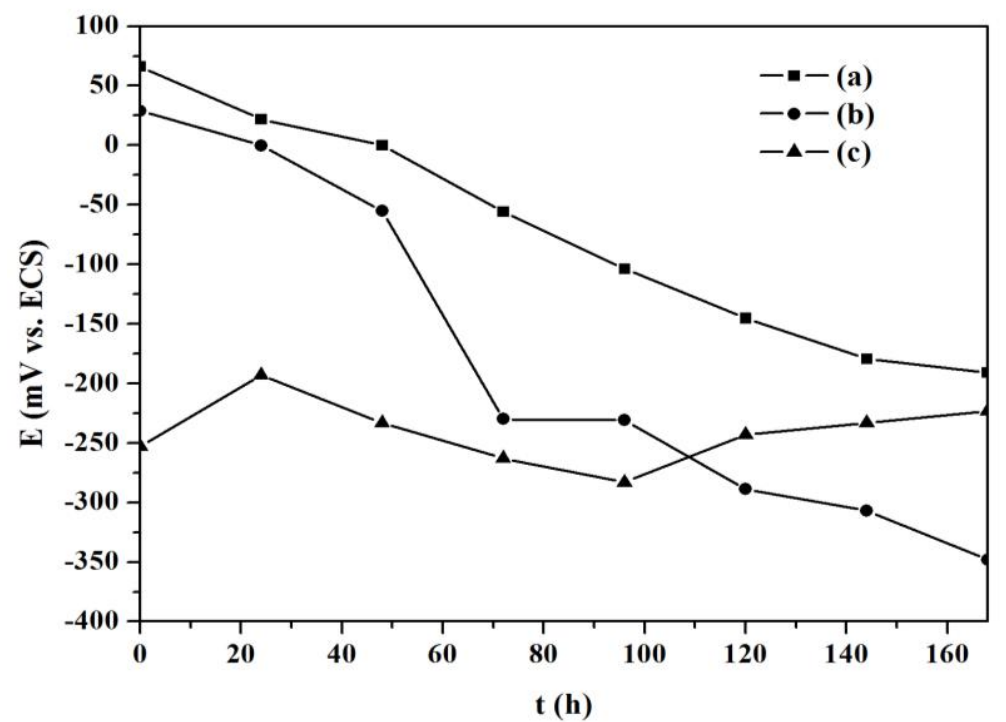

Figura 6: Dependencia del PCA con el tiempo en solución de Ringer durante una semana para: (a) NiTi/PPy ${ }_{1}$, (b) Ni$\mathrm{Ti} / \mathrm{PPy}_{7} \mathrm{y}(\mathrm{c}) \mathrm{NiTi}$ desnudo.

Como se puede observar, para ambas muestras recubiertas el valor inicial del PCA es mucho más positivo que el de la aleación desnuda. La muestra recubierta con $\mathrm{PPy}_{1}$ (Fig. 6, curva a) siempre mantiene un

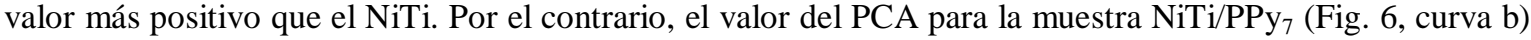
disminuye hasta acercarse al valor correspondiente al de la aleación sin recubrir, lo cual indicaría que el polímero deja de proteger al sustrato.

Luego de esta experiencia, se analizó el contenido de Ni y Ti en la solución de Ringer (Tabla 2). Los datos confirman lo observado en las medidas de PCA. Ambas películas protegen al sustrato, disminuyendo considerablemente la liberación de iones $\mathrm{Ni}^{2+}$, siendo mayor el grado de protección alcanzado en el caso de $\mathrm{PPy}_{1}$.

Tabla 2: Concentración de Ni y Ti liberados luego de una semana a PCA en solución de Ringer para los distintos electrodos recubiertos con PPy y para NiTi desnudo.

\begin{tabular}{l|l|l}
\hline MUESTRA & $\begin{array}{l}\text { CONCENTRACIÓN DE Ni } \\
{\left[\mathbf{m g ~ L}^{-1}\right]}\end{array}$ & $\begin{array}{l}\text { CONCENTRACIÓN DE Ti } \\
{\left[\mathbf{m g ~ L}^{-1}\right]}\end{array}$ \\
\hline $\mathrm{NiTi} / \mathrm{PPy}_{1}$ & 0,77 & $<0,05$ \\
\hline $\mathrm{NiTi} / \mathrm{PPy}_{7}$ & 2,80 & $<0,05$ \\
\hline $\mathrm{NiTi}$ desnudo & 14,96 & $<0,05$ \\
\hline
\end{tabular}

También se llevaron a cabo las pruebas necesarias para confeccionar gráficos de Tafel para los electrodos recubiertos y se compararon con el NiTi desnudo, lo cual se muestra en la Figura 7. Para ésto se polarizaron las muestras a partir de potenciales catódicos hasta potenciales anódicos respecto del PCA. La estimación de los parámetros de corrosión, potencial $\left(\mathrm{E}_{\text {corr }}\right)$ y densidad de corriente $\left(\mathrm{i}_{\text {corr }}\right)$, se obtuvieron a partir del método de extrapolación de Tafel y sus valores se presentan en la Tabla 3. Como se puede observar, el $\mathrm{E}_{\text {corr }}$ es más positivo cuando la aleación está recubierta. En el caso de $\mathrm{i}_{\text {corr }}$ los valores son mayores para las muestras recubiertas. La interpretación de estos datos es más complicada ya que la densidad de corriente no puede ser atribuida solamente a la disolución del metal ya que existe una contribución relacionada a la actividad redox del polímero [21,22]. 


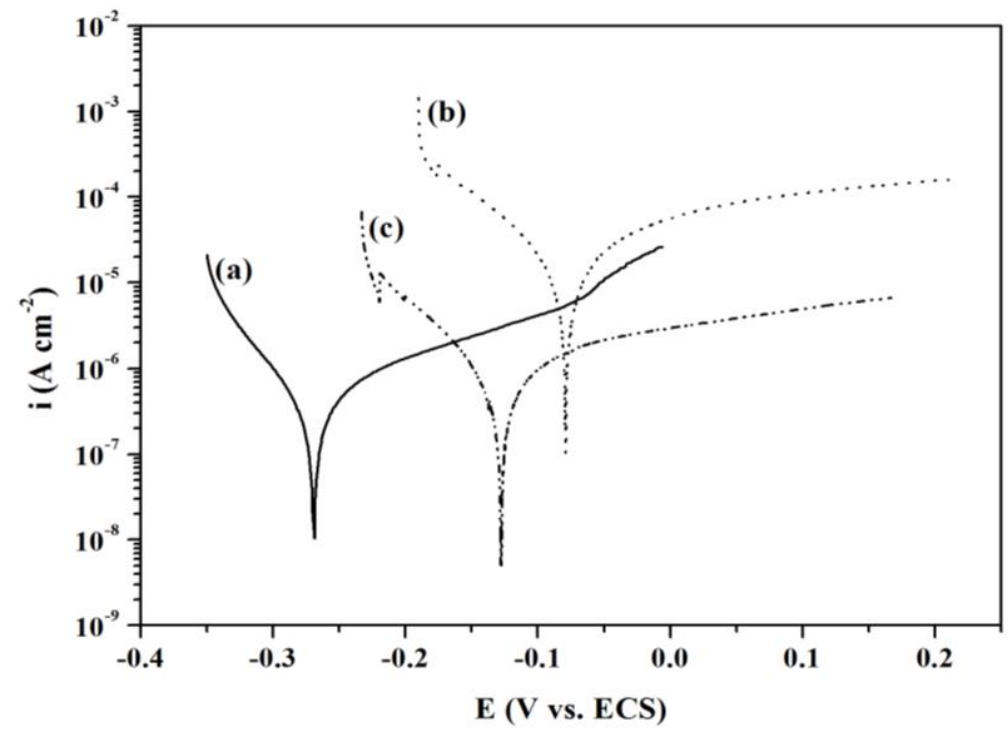

Figura 7: Diagrama de Tafel para (a) NiTi desnudo, (b) NiTi/PPy y (c) NiTi/PPy $y_{7}$ en solución de Ringer. Velocidad de barrido: $5 \mathrm{mV} \mathrm{s}^{-1}$.

Tabla 3: Valores de potencial y densidad de corriente de corrosión obtenidos a partir de los gráficos de Tafel para las distintas muestras.

\begin{tabular}{l|l|l}
\hline MUESTRA & $\begin{array}{l}\mathbf{E}_{\text {CORR }} \\
\text { [V vs. ECS] }\end{array}$ & $\begin{array}{l}\mathbf{i}_{\text {CORR }} \\
{\left[\mathbf{A ~ c m}^{-2}\right]}\end{array}$ \\
\hline $\mathrm{NiTi}^{-} \mathrm{PPy}_{1}$ & $-0,081$ & $1,963 \times 10^{-5}$ \\
\hline $\mathrm{NiTi} / \mathrm{PPy}_{7}$ & $-0,124$ & $5,662 \times 10^{-7}$ \\
\hline NiTi desnudo & $-0,274$ & $3,013 \times 10^{-7}$ \\
\hline
\end{tabular}

La mejora en la resistencia ante la corrosión también puede ser evaluada mediante la comparación de curvas de polarización en el medio agresivo. Para ésto se realizó un barrido potenciodinámico tanto para las muestras recubiertas como para el sustrato desnudo (Fig. 8).

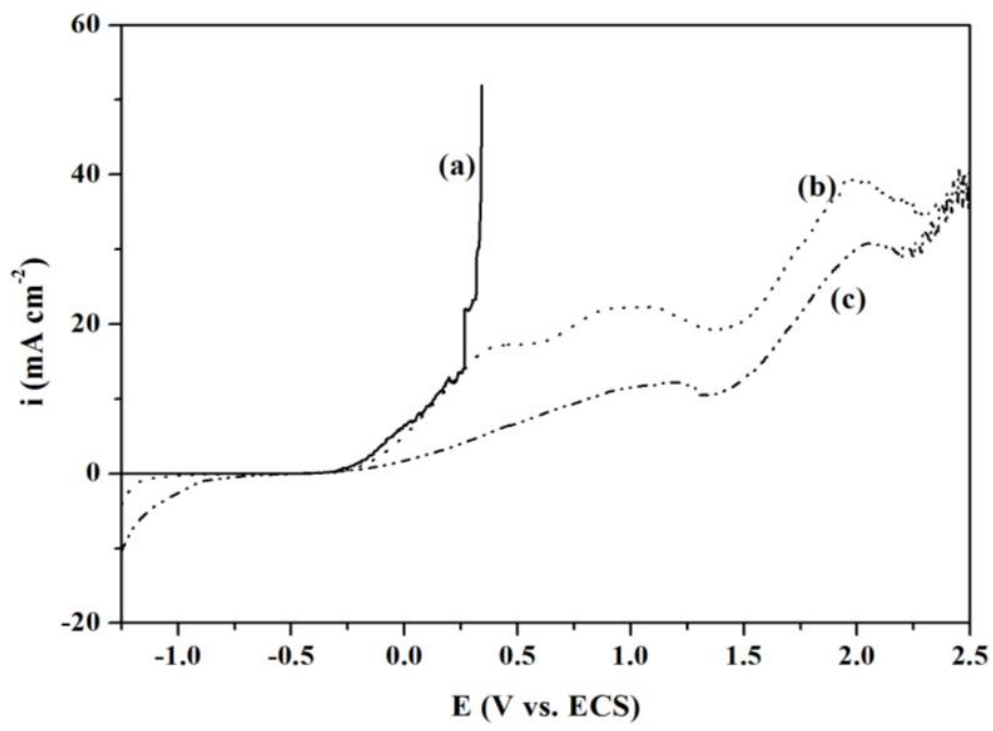

Figura 8: Voltametría de barrido lineal de (a) NiTi desnudo, (b) NiTi/PPy y (c) NiTi/PPy en solución de Ringer. Velocidad de barrido: $5 \mathrm{mV} \mathrm{s}^{-1}$. 
En el caso del NiTi desnudo (Fig. 8, curva a), la corriente aumenta rápidamente luego de la zona pasiva, lo cual indica el comienzo de la corrosión por picado [23]. Para las muestras recubiertas (Fig. 8, curvas b y c), se puede observar una zona pasiva similar a la del NiTi desnudo. Luego, la curva presenta picos anódicos asociados con la oxidación del polímero [24, 25]. A potenciales mayores a 1,0 V(ECS) tienen lugar las reacciones de desprendimiento de oxígeno y cloro, simultáneamente con la sobreoxidación del polímero [26,27]. A potenciales más positivos, el valor de la corriente medida para los electrodos recubiertos comienza a oscilar, lo cual indica el comienzo de la corrosión por picado. Estos resultados indican que al recubrir el sustrato se alcanza una mayor protección ante la corrosión por picado.

\section{CONCLUSIONES}

En este trabajo se presentan los resultados sobre la electropolimerización de PPy sobre Nitinol, a partir de soluciones de distinto $\mathrm{pH}$ ( 1 y 7) que contienen el monómero y ácido sulfosuccínico. Las muestras se formaron potenciostáticamente, formando películas de igual espesor. Se lograron obtener capas de polímero gruesas y compactas. Las medidas de fuerza de adherencia demuestran que aquellas capas de polímero formadas a pH 1 son mucho más adherentes que las formadas a pH 7. Para determinar el grado de protección que brinda el polímero se realizaron distintas pruebas electroquímicas en solución de Ringer. Los barridos potenciodinámicos demuestran que ambos recubrimientos protegen al sustrato de la corrosión por picado. Los resultados obtenidos a partir de las medidas de PCA y Tafel indican que las dos películas formadas ( $\mathrm{pH} 1$ y $\mathrm{pH} 7)$ brindan protección al NiTi. Sin embargo, comparando el comportamiento de ambas, la sintetizada a pH 1 ofrece una mayor protección, lo cual fue confirmado mediante el análisis de iones Ni y Ti disueltos en solución.

\section{AGRADECIMIENTOS}

Al aporte financiero realizado por la Secretaría de Ciencia y Técnica - UNS (PGI 24/M146), al Consejo Nacional de Investigaciones Científicas y Técnicas (CONICET- PIP 112-20150-100147) y a la Agencia Nacional de Promoción Científica y Tecnológica (ANPCYT PICT-2012-0141).

\section{BIBLIOGRAFÍA}

[1] ANDANI, M.T., MOGHADDAM, N.S., HABERLAND, C., DEAN, D., MILLER, M.J., ELAHINIA, M., "Metals for bone implants. Part 1. Powder metallurgy and implant rendering", Acta Biomaterialia, v. 10, n. 10, pp. 4058-4070, October 2014.

[2] LIU, X, CHU, P.K., DING, C., "Surface modification of titanium, titanium alloys, and related materials for biomedical applications", Materials Science and Engeneering R, v. 47, n. 3-4, pp. 49-121, December 2004.

[3] OLLER, A.R., COSTA, M., OBERDORSTER, G., "Carcinogenicity assessment of selected nickel compounds", Toxicology and Applied Pharmacology., v. 66, n. 1, pp. 143-152, March 1997.

[4] TALLMAN, D.E., DEWALD, M.P., VANG, C.K., et al., "Electrodeposition of conducting polymers on active metals by electron transfer mediation", Current Applied Physics, v. 4, n. 2-4, pp. 137-140, April 2004.

[5] GUIMARD, N.K., GOMEZ, N., SCHMIDT, C.E., "Conducting polymers in biomedical engineering", Progress in Polymer Science, v. 32, n. 8-9, pp. 876-921, August-September 2007.

[6] RYU, H., SHENG, N., OTHTSUKA, T., et al., "Polypyrrole film on 55\% Al-Zn-coated steel for corrosion prevention”, Corrosion Science, v. 56, n. 3, pp. 67-77, March 2012.

[7] GRUBAČ, ŠKUGORONČEVIĆ, I., METIKOŠ-HUKOVIĆ, M., "Corrosion protection of aluminum by electrochemically synthesized composite organic coating, Corrosion Science, v. 102, n. 1, pp. 310-316, January 2016.

[8] MERT, B.D., "Corrosion protection of aluminum by electrochemically synthesized composite organic coating”, Corrosion Science, v. 103, n. 2, pp. 88-94, February 2016.

[9] IDLA, K., INGANÄ, O., STRANDBERG, M., "Good adhesion between chemically oxidised titanium and electrochemically deposited polypyrrole", Electrochimica Acta, v. 45, n. 13, pp. 2121-2130, March 2000.

[10] DUFOUR, B., RANNOU, P., DJURADO, D., et al., "Low Tg, Stretchable, Polyaniline of Metallic-Type Conductivity: Role of Dopant Engineering in the Control of Polymer Supramolecular Organization and in the Tuning of Its Properties", Chemistry of Materials, v. 15, n. 8, pp. 1587-1592, August 2003. 
[11] BOUKERMA, K., OMASTOVÁ, M., FEDORKO, P., et al., "Surface properties and conductivity of bis(2-ethylhexyl) sulfosuccinate-containing polypyrrole", Applied Surface Science, v. 249, n. 1-4, pp. 303314, August 2005.

[12] LEHR, I.L., SAIDMAN, S.B., "Corrosion protection of iron by polypyrrole coatings electrosynthesised from a surfactant solution", Corrosion Science, v. 49, n. 5, pp. 2210-2225, May 2007.

[13] FLAMINI, D.O., SAIDMAN, S.B., "Characterization of polypyrrole films electrosynthesized onto titanium in the presence of sodium bis (2-ethylhexyl) sulfosuccinate (AOT)", Electrochimica Acta, v. 55, n. 11, pp. 3727-3733, April 2010.

[14] BURSTEIN, G.T., LIU, C., SOUTO, R.M., "The effect of temperature on the nucleation of corrosion pits on titanium in Ringer's physiological solution", Biomaterials, v. 26, n. 3, pp. 245-256, January 2005.

[15] PEI, Q., QIAN, R., "Electrode potentials of electronically conducting polymer polypyrrole", Electrochimica Acta, v. 37, n. 6, pp. 1075-1081, May 1992.

[16] MÎNDROIU, M., UNGUREANU, C., ION, R., et al., "The effect of deposition electrolyte on polypyrrole surface interaction with biological environment", Applied Surface Science, v. 276, pp. 401-410, March 2013.

[17] FLAMINI, D.O., SAUGO, M., SAIDMAN, S.B., "Electrodeposition of polypyrrole on Nitinol alloy in the presence of inhibitor ions for corrosion protection", Corrosion Science, v. 81, n. 4, pp. 36-44, April 2014.

[18] YAKUPHANOGLU, F., YAHIA, I.S., SENKAL, B.F., et al., "Impedance spectroscopy properties of polypyrrole doped with boric acid", Synthetic Metals, v. 161, n. 9-10, pp. 817-822, May 2011.

[19] BAI, Y., XU, Y., WANG, J., et al., "Interface effect on the electropolymerized polypyrrole films with hollow micro/nanohorn arrays", Applied Materials and Interfaces, 2014, v. 6, n. 7, pp 4693-4704, March 2014.

[20] GRARI, O., DHOUIBI, L., LALLEMAND, F., et al., "Effects of nitrate ions on the electrochemical synthesis and behavior of polypyrrole films", Progress in Organic Coatings, v. 77, pp. 1867-1873, July 2014.

[21] ANNIBALDI, V., ROONEY, A.D., BRESLIN, C.B., "Corrosion protection of copper using polypyrrole electrosynthesised from a salicylate solution", Corrosion Science, v. 59, n. 6, pp. 179-185, June 2012.

[22] FLAMINI, D.O., SAIDMAN, S.B., "Electrodeposition of polypyrrole onto NiTi and the corrosion behaviour of the coated alloy", Corrosion Science, v. 52, n. 1, pp. 229-234, January 2010.

[23] MAN, H.C., HO, K.L., CUI, Z.D., "Laser surface alloying of NiTi shape memory alloy with Mo for hardness improvement and reduction of Ni2+ ion release", Surface \& Coatings Technology, v. 200, pp. 4612 - 4618, June 2005.

[24] EL JAOUHARI, A., EL ASBAHANI, A., BOUABDALlAOUI, M., et al., "Corrosion resistance and antibacterial activity of electrosynthesized polypyrrole", Synthetic Metals, v. 226, pp. 15-24, January 2017.

[25] OTERO, T.F., ARIAS-PARDILLA, J., CHERMAK, E., "Reactive polymer films. Polypyrrole oxidation kinetics in aqueous solution", Synthetic Metals, v. 160, pp. 425-431, January 2010

[26] TÜKEN, T, "Polypyrrole films on stainless steel", Surface and Coating Technology, v. 200, n. 16-17, pp. 4713-4719, April 2006.

[27] QI, K., QIU, Y., CHEN, Z., et al., "Corrosion of conductive polypyrrole: Effects of continuous cathodic and anodic polarisation", Corrosion Science, v. 69, n. 4, pp. 376-388, April 2013. 